\title{
Snapshot of HIV pathogenesis in China
}

\author{
Nitin K SAKSENA ${ }^{1, *}$, Bin WANG ${ }^{1}$, Megan STEAIN ${ }^{1}$ Rong Ge YANG ${ }^{2}$, Lin Qi ZHANG ${ }^{3,4,5}$ \\ ${ }^{1}$ Retroviral Genetics Division, Centre for Virus Research, Westmead Millennium Institute, The University of Sydney, Westmead \\ NSW 2145 Sydney, Australia \\ ${ }^{2}$ AIDS and HIV Research Group, Wuhan Institute of Virology, Chinese Academy of Sciences, Wuhan 430071, China \\ ${ }^{3}$ Aaron Diamond AIDS Research Center, The Rockefeller University, New York, NY 10021, USA \\ ${ }^{4}$ Modern Virology Research Center and AIDS Center, State Key Laboratory of Virology, College of Life Sciences, Wuhan \\ University, Wuhan 430072, China \\ ${ }^{5}$ The AIDS Research Center, Chinese Academy of Medical Sciences, Peking Union Medical School, Beijing 100730, China
}

\begin{abstract}
Several reviews have focused on the nature of HIV infection and its spread in various geographical regions of China. In contrast, this review provides a comprehensive update on the prevalence of multiple HIV-1 subtypes, consequent emergence of recombinant and novel forms of HIV-1 in China, and the implications this may have on HIV diversity and the development of effective vaccines. In addition it also examines the dissemination of primary drug resistance in therapy naïve patients, as well as co-infections with two other important viruses-hepatitis B and C. The main purpose of this review is to provide a current snapshot of HIV-1 pathogenesis in China and possibly shed some light on the future of HIV evolution, and potential challenges for future vaccine and anti-retroviral therapeutics against HIV strains in this area.
\end{abstract}

Keywords: HIV, recombination, circulating recombinant forms, co-infections, AIDS.

\section{INTRODUCTION}

A recent report by the National Intelligence Council estimates that by 2010 five countries, India, China, Nigeria, Ethiopia and Russia, cumulatively, will harbor the largest number of individuals infected with the Human Immunodeficiency Virus (HIV) in the world [1]. These countries are of immense strategic importance to the United States, and economically, India and China remain among the most lucrative countries to rest of the world. The spread of HIV, and the associated morbidity and mortality, may affect the socio-economic potential of these countries, due to a decrease in the size of the workforce and the costs associated with management of disease. In the future, HIV/ AIDS may be the single most important factor in defining the very balance of power within Eurasia and its relationship with rest of the world.

\footnotetext{
*Correspondence: Nitin K SAKSENA

Tel: 612-98459119; Fax: 612-88243098;

E-mail: Nitin_saksena@wmi.usyd.edu.au.
}

Since the main focus of this review is China, it is appropriate to say that the continuing economic growth and prosperity of China will, in the coming decades, be defined by the spread of killer diseases, especially HIV. Thus to grow and prosper, the spread of infectious agents will have to be contained through strict measures. Only then can the socio-economic fabric of the society be kept intact and prosperity sustained.

China is facing an increasing danger of a widespread HIV-1 epidemic in high-risk populations such as injecting drug users (IDU) and paid blood donors (PBD), and of further dissemination from these high risk populations to the general public. The virus has spread to every province and autonomous region, and the government estimates about 840,000 infections, of which only 89,067 have been reported [2]. However, prevalence and incidence studies are woefully inadequate and the United Nations Joint Program on HIV/AIDS estimates that there may be as many as 10 million infected people by $2010[1,2]$. Over the last couple of years, increasing efforts from the Chinese 
government, non-government and international organizations in carrying out various preventive measures aiming to raise the awareness and improve the knowledge of this disease have been initiated. In March, 2003, the Chinese government began implementing a free national ARV treatment program. However, its reach is limited due to an insufficient number of properly trained physicians, nurses and community care workers. The majority of infected patients, particularly IDUs, do not have access to care and for those that do, drug regimens are limited. Therefore, HIV continues to spread rapidly in China despite increased openness by health officials and increased preventive and therapeutic measures.

The first cases of HIV infection in China were reported in the year 1985 in four Chinese hemophiliacs whom had received blood products from the United States [3]. Then from 1989 a number of infections arose in Chinese intravenous drug users from the Yunnan Province [4-7] and it appears this may be the point of common origin of the present day HIV epidemic in China. Thus the 2 decades of HIV prevalence in China was divided into three phases [8, 9].

Between 1985 and 1988 the first phase occurred and comprised of only of small numbers of imported cases originating from expatriate Chinese or the foreigners visiting China as a result of considerable economic growth. These cases appeared sporadically, mainly around the coastal regions of China [10]. Four Chinese individuals with hemophilia from the Zhejiang province were also infected after receiving an imported factor VIII concentrate contaminated with HIV.

Most of the second and third phases of the HIV epidemic can largely be correlated with the drug trafficking routes that exist between and across China from Myanmar (Burma) and Laos. A 'golden triangle' of drug producing regions exists in this area and comprises of northern Thailand, northern and eastern Myanmar (Myanmar produces $60 \%$ of the world's heroin supplies) and western Laos (the third leading producer of heroin) [11].

The second phase of the epidemic occurred between 1989 and 1993. Around 1989, 146 HIV positive injecting drug users were identified in southwest of the Yunnan Province $[4,6-8,12]$. During this period the large majority of all HIV cases identified in China were from this region. The principal reason for these infections is that the Yunnan province shares a common border with Myanmar (Burma) which forms part of a major trafficking route for heroin into China [11]. Almost all of the identified cases of HIV in this area were in intravenous drug users or their partners [11]. However, the high prevalence of HIV infection that was seen decreased as the distance to from the Chinese-Burmese border increased [11]. But the current trends of HIV spread show HIV prevalence in Yunnan to be $25 \%$, whereas in Xinjiang it is as high as $70 \%$.

The third, and the most complex phase of HIV epidemic in China, began in 1994. During this phase HIV was beginning to be identified in many provinces of China, and not just in IDUs. This phase has so far been characterized by a myriad of HIV strains, multiple subtypes, circulating recombinant variants and the emergence of drug resistant strains, which together have changed the complexion of the HIV epidemic in China and its surrounding geographical regions. During this ongoing third phase, a large number of HIV infections have again been observed among drug users from provincial regions of China that are along heroin trafficking routes (Yunnan, Xinjiang, Sichuan and Guangxi) [7]. Needle sharing is commonly practiced among drug traders as well as users along the routes of heroin trafficking. Coinciding with the IDU spread of HIV, the heterosexual spread of HIV has also increased, but has not reached epidemic proportions as seen with the IDU-related spread $[8,9]$. In addition a second cluster of HIV infected individuals have also been identified in Henan and surrounding provinces such as Hubei, Shanxi and Anhui. It was discovered that companies that were paying individuals for donating blood, were collecting plasma and then the red blood cells of numerous individuals were pooled and injected back into the donors [13]. Such practices, in addition to the re-use of needles and unsterilized equipment, have given the virus an easy and rapid route of transmission to the local population. It is now believed that in the mid to late 1990's, a large number of people in Central China became HIV infected by selling their blood under such conditions. This then lead to an outbreak of HIV infections, with a single HIV subtype B' [14], in rural villages where people were using this practice to supplement their income.

Seeing the various trends since the onset of HIV epidemic in China, it is plausible to say that there is also the fourth ongoing phase in Chinese HIV epidemic, which should start in March 2003 coinciding with the introduction of a national free anti-retroviral (ARV) treatment program in China. The start of this phase reflects government's strong commitment to treat this disease and improve the care and standard of those infected. The mortality rate has dropped considerably and in many places, the quality of life has been greatly improved. However, the same ARV treatment program has caused many unforeseen problems during this massive emergency operation.

In many areas where ARV treatment was initiated, there were insufficient number of properly trained doctors, nurses, and community care workers to provide standard care on a daily basis and a lack of sufficient testing proce- 
dures to monitor the treatment efficacy. In addition, severe side effects associated with generic ARV produced in China led to a large number of patients either quitting medication entirely or missing doses frequently. Such incomplete care services combined with inconsistent compliance have allowed HIV-1 a unique opportunity to evade ARV drugs through the emergence of genetic variants that resist different drugs within the regimen. To make things worse, there is limited number of ARV drugs in China, and some generic ARV drugs made locally are in sub-optimal formation due to patent protection issues. This has left many patients with no choice but to live with the virus or resort to obtaining drugs in the black market. This will inevitably cost their lives within a predictable time frame. Thus, the third and fourth phases are ongoing and are highlighted by the emergence and transmission of drugresistant strains, continuous spread of multiple genotypes as well as increasing proportion of recombinant forms as a result of multiple genotypes circulating among the same high-risk groups in the same region.

Now HIV infections have been reported from all 31 provinces of China including various municipalities and autonomous regions [13]. This review provides an overview of HIV pathogenesis mainly taking into account the ongoing third and fourth phases of the HIV epidemic in China. In addition, it also discusses various aspects and problems related to HIV co-infections, recombination and the emergence and dispersal of drug resistance. These aspects are critical and may have important biological implications as far as HIV pathogenesis is concerned in the most populous nation of the world.

\section{HIV RECOMBINATION IN CHINA AND SUR- ROUNDING REGIONS: IT'S ROLE IN TRANS- MISSION AND PATHOGENESIS}

One of the unique traits of the human immunodeficiency virus (HIV) is its ability to generate multiple genetic variants, which may facilitate the immune evasion of HIV. Recombination is one such strategy the virus employs to generate genetically diverse, fitter and possibly more virulent forms. Therefore, the recombinant forms of HIV-1 are believed to harbor altered cytopathic and/or pathogenic potential, but the biological proof to attest this belief is lacking. Some recombinant HIV-1 strains have disseminated widely in populations, leading to their definition of "circulating recombinant forms" (CRFs) [15]. These CRFs are playing a major role in the global and regional HIV epidemics, especially in regions where multiple subtypes are circulating.

Sixteen CRFs are currently recognized (http://hiv-web. lanl.gov/CRFs/CRFs.html) (Table 1). Of these 16, three CRFs appeared to have played a critical role in the HIV-1
Tab. 1 List of circulating recombinant forms identified globally

\begin{tabular}{lll}
\hline Name & Reference strain & Subtypes \\
\hline CRF01_AE* & CM240 & A, E \\
CRF02_AG & IbNG & A, G \\
CRF03_AB* & Kal153 & A, B \\
CRF04_cpx & 94 CY032 & A, G, H, K, U \\
CRF05_DF & VI1310 & D, F \\
CRF06_cpx & BFP90 & A, G, J, K \\
CRF07_BC* & CN54 & B', C \\
CRF08_BC* & GX-6F & B', C \\
CRF09_cpx & 96 GH2911 & not yet published \\
CRF10_CD & TZBF061 & C, D \\
CRF11_cpx & GR17 & A, CRF01_AE, G, J \\
CRF12_BF & ARMA159 & B, F \\
CRF13_cpx & $96 C M-1849$ & A, CRF01_AE, G, J, U \\
CRF14_BG & X397 & B, G \\
CRF15_01B & $99 T H . M U 2079$ & CRF01_AE, B \\
CRF16_A2D & KISII5009 & A2, D \\
\hline
\end{tabular}

* denote CRFs circulating in China

epidemic in Asia. Notably, CRF01_AE, which was originally identified in Thailand [16], has spread throughout SEA, including China [17].

From the comparison of the present and previous HIVepidemic in China, it is apparent that rapid dissemination and establishment of certain recombinant viral strains may be gradually phasing-out pure HIV-1 subtypes. Other South East Asian (SEA) countries, proximal to China, have also experienced similar epidemiological shifts, for example Thailand [18]. China is also no exception to this phenomenon and currently the Chinese HIV epidemic is dominated by high prevalence of a variety CRFs [7, 19].

It has been reported that the HIV-1 epidemic among IDUs in Yunnan Province was initiated in late 1980s by HIV-1 subtype B (American/European lineage) in addition to HIV-1 subtype B' (referred to as Thai-B) strains [17, 20]. Interestingly, subtype $B$ ' actively replaced the subtype B strains of American lineage, and increased from $20 \%$ in 1990 to $90 \%$ in 1996 [19]. Following this, HIV-1 subtype C strains were also identified in early 1990s among IDUs [21]. As a result of the co-circulation of these two subtypes, $\mathrm{B}$ and $\mathrm{C}$, two CRFs arose, most likely in the Yunnan province, CRF07_BC and CRF08_BC. These were first identified among injecting drug users from across China (IDUs) [4, 22].

CRF07_BC (originated from prototypic strains 97CN54 and $97 \mathrm{CN001)}$ is distributed mainly among IDUs in the Province of Xinjiang situated in northwest of China [22]. In contrast, the CRF08_BC (originating from prototypic 


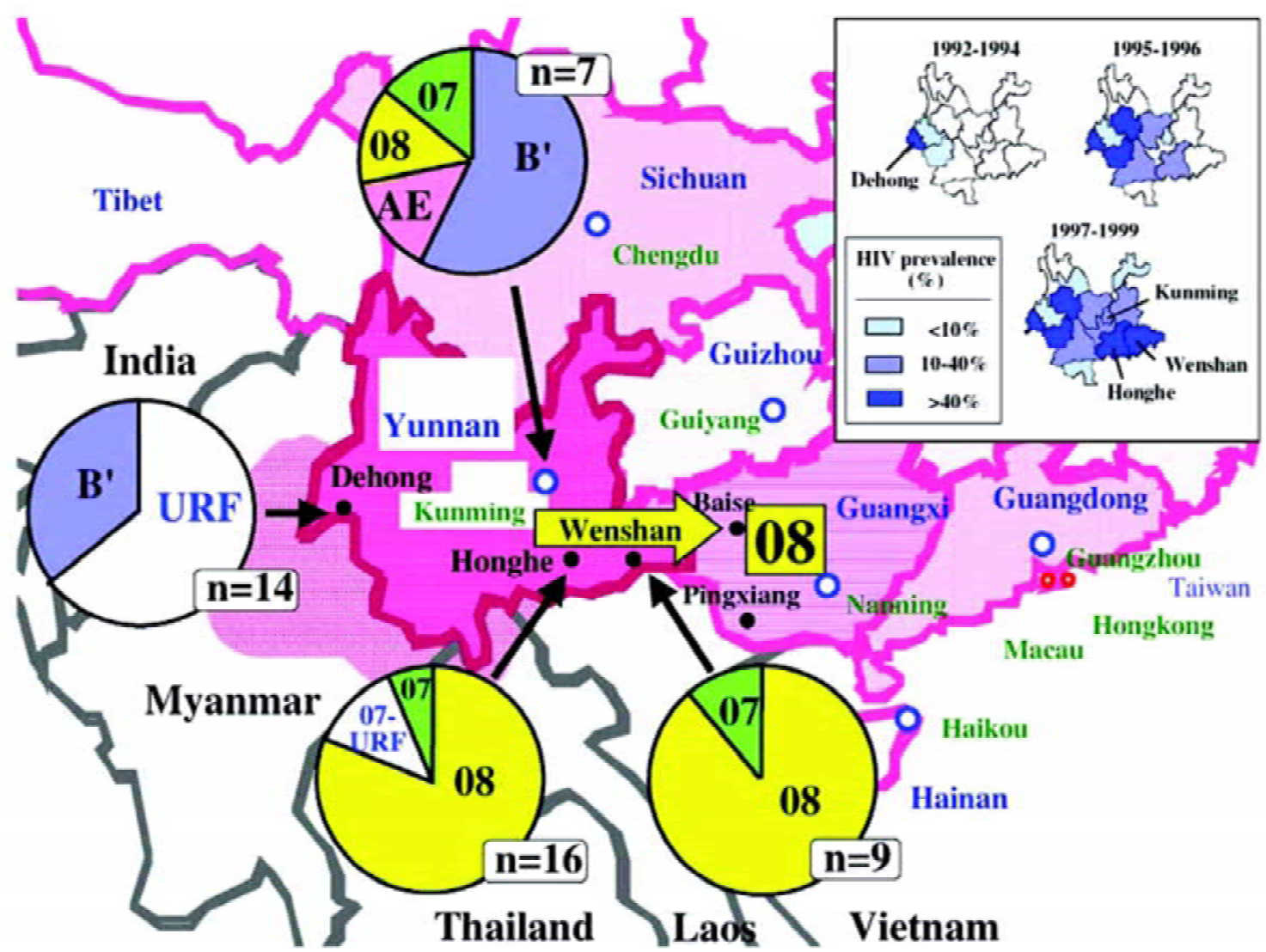

Fig. 1 Geographical distribution of HIV-1 subtypes, CRFs and URFs among IDUs, in addition to trends in yearly increase of HIV1 prevalence rates in different locales of Yunnan province, China. Each pie shows the breakdown of prevalence of individual subtypes, URFs and CRFs in various geographical locales used in this study (indicated by closed arrows). Open arrows indicate the possible route of spread of CRF08_BC that is known to be the predominant circulating strain among IDUs in Guanxi Province. The checkered region shows the "Golden Triangle", a major drug trading area bordering Thailand, Myanmar and Laos. The inset shows the temporal and spatial changes in HIV-1 prevalence rates among IDU in Yunnan province, with the sites of study indicated in the map (Wenshan, Honghe, and Dehong). The data for Kunming are based on the archival samples, collected between 1994-1997 from HIV+ and sexually transmitted disease patients.

strain, 97CNGX6F) has been observed circulating among IDUs in the province of Guangxi in southeast of China [4]. As heroin trafficking is highly prevalent, notable is that the distribution and dissemination of these CRFs appears to bear association with a different heroin trafficking routes. For instance, both CRF07_BC and CRF08_BC presumably originated in the Yunnan Province (both subtypes B and C co-circulate in this Province), but have followed different patterns of geographical spread, with CRF07_BC spreading northwestward to Xinjiang and CRF08_BC eastward to Guangxi Provinces, respectively [4] (Fig. 1). Furthermore, dissemination of CRF07_BC has recently been found to IDU from Taiwan (Hsi-Hsun Lin, Section of Infectious Diseases, Department of Medicine, E-Da Hospital/I-Shou University, personal communication).
These two CRFs continue to co-circulate with pure subtypes B' and C, as well as with unique recombinant forms in Yunnan [7]. Consequently new recombinant strains between these two CRFs have also been described [19], highlighting the ongoing evolution of strains in this region, which will make vaccine design progressively more complicated. The second and third generation recombinant CRFs in China harbor extensive variability in breakpoints, making vaccine design even more difficult. One such example of genetic diversity is shown in Fig. 2, where variability in profiles of crossover points between HIV CRF07_BC and CRF08_BC are shown. This is the first example of recombination between CRFs, which may have immense biological implications at the level of virulence, efficient transmission, and the virus acquiring fitness. 


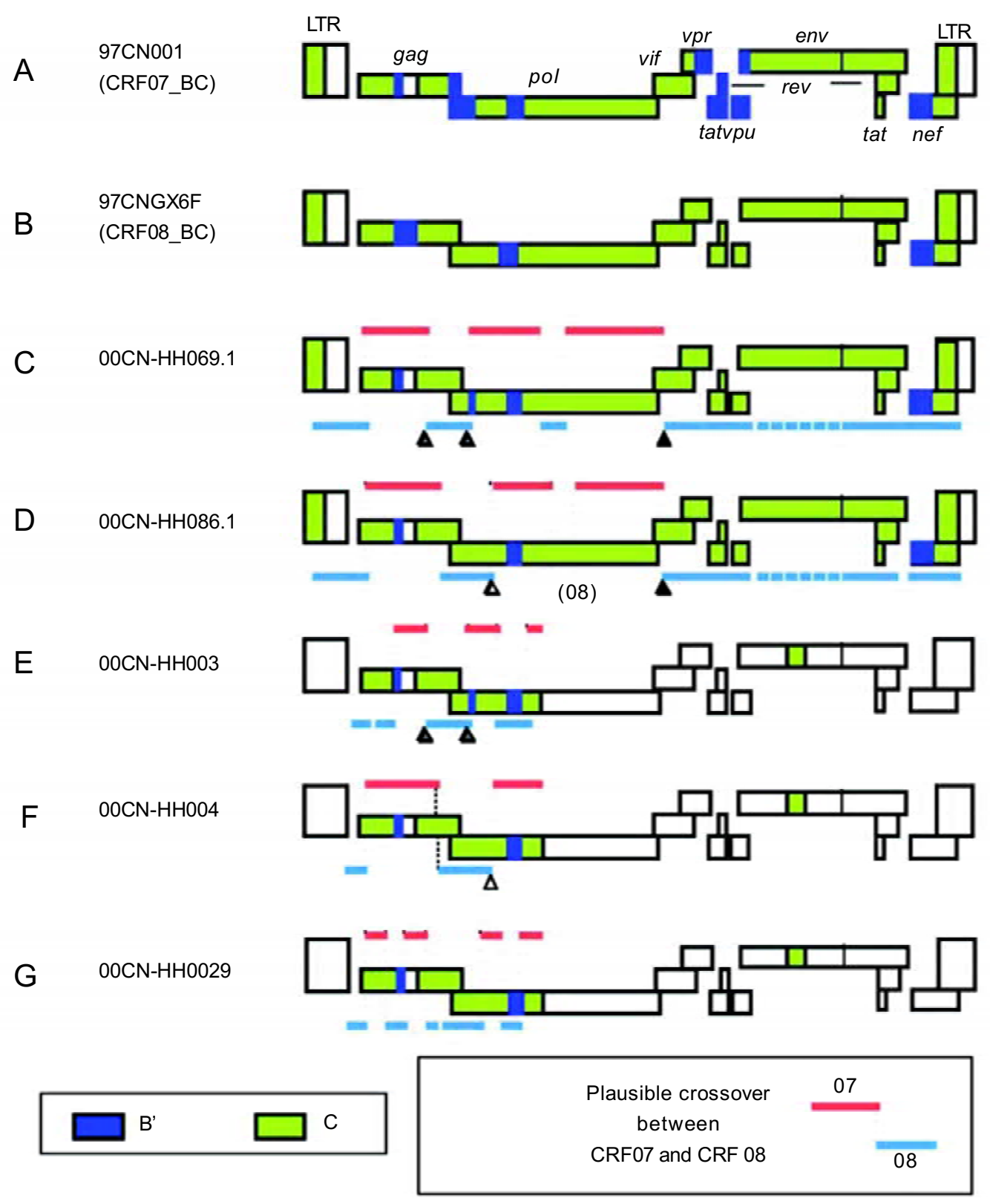

Fig.2 Diagrammatic representation of molecular architecture of subtype and deduced profiles of crossover points between HIV CRF07_BC and CRF08_BC in new "second-generation" inter-CRF recombinants identified in Honghe Prefecture of Yunnan Province, China. This is the first demonstration showing that CRFs can further recombine to produce complex mosaic forms of HIV.

HBV AND HCV INFECTIONS AND CO-INFECTIONS

Viral infections spreading through the parenteral route, especially HIV, hepatitis B and hepatitis C viruses, have increased dramatically, affecting regional populations in the Yunnan and Guangdong provinces of China. This is mainly attributed to sharing of contaminated syringes with infected individuals. Together intravenous drug use and paid sex has been recognized as the key drivers of epidemiologic spread of HIV and hepatitis viruses in China [23]. China accounts for one third of chronic HBV and about one quarter of chronic HCV infections worldwide. In total 
China has more than 20 million individuals suffering from viral liver diseases. There are approximately 350 million carriers of HBV and more than 40 million infected (chronic sufferers) worldwide [24] and Joint UN Program www. unaids.org (2005). In China alone, 250-280,000 HBV related deaths occur annually, and it remains the biggest killer infectious agent in China. In the prospective US study, the Multicenter AIDS Cohort study (MACS), $6 \%$ of men who have sex with men [25] and $10 \%$ of injection drug users with HIV infection were co-infected with HBV [2628]. It has therefore been suggested that patients infected with HIV should also be screened for hepatitis A, B and C viruses. In some parts of China there has been a rapid upsurge in cases of HIV-1 and hepatitis C virus (HCV) co-infections [29-31]. This pattern of dissemination is mainly attributed to paid blood donors (PBD), injecting drug users (IDU) and their sexual partners, along with undiagnosed HIV positive individuals. These HIV/HCV and/ or HBV co-infections have become the main cause of morbidity and mortality in immuno-compromized HIV-infected patients.

In some provinces of central China, as indicated above, a number of HIV infections are concentrated among commercial plasma donors. Some illegal commercial companies provide plasma by recruitment of individuals from a very mobile and unscreened population who are known to engage in prostitution and drug abuse. These are the individuals who pose the biggest risk for HIV and Hepatitis virus transmission and spread of co-infections.

Recently, Zhang et al [29] analyzed 236 HIV-1 positive blood samples collected from PBD, IDU, and their sexual partners hailing from the provinces of Henan, Yunnan, Guangxi, and Xinjiang, in China. These provinces are some of the worst affected by HIV-1. In this study, Zhang et al. [29] amplified and sequenced the p17 region of the gag gene and $\mathrm{C} 2-\mathrm{V} 3$ region of the env gene of HIV- 1 and the 5 ' non-coding region and a region of E1/E2 of HCV. Together the epidemiologic and molecular data provide evidence in favor of two major epidemics of HIV-1 as opposed to multiple HCV epidemics in China. Both PBD and transfusion recipients from the Henan province harbored HIV-1 subtype B', similar strains of Thailand origin, and $\mathrm{HCV}$ genotypes were $1 \mathrm{~b}$ and 2a. In contrast, the scenario was different among IDUs from Yunnan, Guangxi, and Xinjiang provinces where HIV-infected individuals carried the circulating recombinant forms CRF-07 and CRF-08, which have been previously reported from China and India, and HCV genotypes $1 \mathrm{~b}, 3 \mathrm{a}$, and $3 \mathrm{~b}$. These differences between PBD and IDUs indicate a relationship between HIV-1 subtypes and HCV genotypes and the mode of transmission, rather than geographical area. Although the biological reasons for such interesting disparity are unclear, more epidemiologic data from China and the surrounding regions may clarify this. Overall, from these studies it is clear that these HIV/HCV infections in China are the consequence of multiple introductions. Another study by Zhang $\mathrm{M}$ [32] examined the frequency of HIV and HCV among residents of Linxian (Henan Province). Of 500 participants, $48(9.6 \%)$ were positive for HCV by enzyme immunoassay and recombinant immunoblot assay. For HIV, 0 of 200 were positive, thereby suggesting that although HIV and $\mathrm{HCV}$ infections and co-infections are on the rise, the $\mathrm{HCV}$ still remains the more common infection among adults in China.

\section{ANTI-HIV THERAPY, DRUG RESISTANCE AND ITS DISSEMINATION IN CHINA}

In global terms, over the past 15 years the treatment of HIV-1 infection has evolved significantly. In North America and Western Europe, no effective therapy existed until the development and availability of zidovudine (ZDV, AZT) in 1987. In 2005, there are now 26 commercially available antiviral agents (both RT inhibitors [NRTI and NNRTI] and protease inhibitors) to treat HIV-1-infected individuals.

ARV treatment of HIV-1-infected patients in China fell behind that of most developed countries. While highly active antiretroviral therapy (HAART) became widely used in North America and Western Europe in 1996, China was still debating whether or not HIV/AIDS would become a huge epidemic there, despite the large number of IDUs testing positive in the southwest province of Yunnan and almost all provinces reporting HIV cases. In 1998, facing the rapid upsurge in HIV-1 incidence nation-wide, the Chinese government made a concerted effort to strategize the "Middle and Long-term Programming for the Prevention and Control of AIDS" in China. A year later in 1999, several small clinical trails were initiated in Beijing primarily for safety and efficacy testing, sponsored largely by international pharmaceutical companies. The drug regimen tested then consisted of Combivir plus either Indinavir or Abacavir. This small-scale trial period (1999-2001) can be regarded as the first phase of ARV treatment in China.

The second treatment phase started when the cost of imported drugs used for HAART declined significantly and more patients could afford the medications (2001-2003). The population of Chinese patients undergoing therapy for HIV increased, especially in economically developed areas such as Beijing and Shanghai. However, the number of clinical doctors trained to administer these drugs did not expand. Many patients did not have the opportunity to receive comprehensive care, including standardized immunologic and virologic assessments prior to treatment and regularly scheduled follow-up interviews. Some patients judged the efficacy of the medication by a moderation of 
their symptoms, and consequently decreased their dosage or stopped taking the medicine altogether, without the consent of a physician. Of the patients who initiated treatment in this period, an estimated 25-30\% stopped taking medicine after only one or two months. Whether or not the other patients were able to persist with treatment and return for follow-up interviews is still to be determined.

The third phase of treatment (2003-present) began with the availability of low-priced domestically manufactured and imported generic anti-HIV drugs. This has been undeniably the most beneficial phase in increasing the number of individuals receiving gratis treatment. Nation-wide free ARV treatment started in 2003, part of the China CARES program, consisting of 51 model sites with plans to further expand to 127 counties. However, the bigger hurdle for this ambitious plan has been again the critical shortage of properly trained doctors, nurses and community care workers. Some patients were so anxious to begin taking medicine for HIV that they obtained the necessary drugs without a doctor's prescription. As a consequence, lacking professional guidance and clinical supervision, they used the medicines improperly, leading to the development of a drug resistant virus. In addition, as generic HIV drugs entered the Chinese market from developing countries, some patients began taking medicine without any medical assessment before treatment, and without choosing to register for interviews during treatment. Furthermore, severe side effects associated with generic ARV produced in China led to a large number of patients stopping medication entirely or becoming unwilling to follow doctors' advice and suggestions. As the incidence of HIV infection rising in China, it is anticipated that problems associated with the abuse of ARV will only escalate. It is therefore expected that drug resistant HIV-1 strains will emerge leading to their high prevalence and transmission over time.

A number of studies in other countries have shown that the prevalence of viruses with drug resistance mutations in acutely or recently infected persons varies from 10 to $20 \%$ [33-35]. Research examining the prevalence and genetic features of drug-resistance strains at national level is lacking in China. Several major institutes in China are combining forces to carry-out genetic studies on viruses collected before and after nation-wide free ARV treatment. Based on preliminary data, it is fairly clear that the prevalence of drug-resistant strains were extremely rare before 2000. Between 2001 and 2003, however, drug-resistant strains began to emerge and in some areas the prevalence is as high as $5 \%$. Beginning in 2004, there was a significant increase in the prevalence of drug-resistant strains across entire China, coinciding with the nation-wide free ARV treatment. Some areas have reported $20-30 \%$ drugresistant strains specifically against NNRTI (unpublished data), and some areas were reported to have as high as $60 \%$ drug-resistant strains. The significant increase in the prevalence of drug-resistance could be due to the selection of the cohort and the time from transmission to resistance testing. However, it has clearly shown that resistance tests should be recommended routinely for patients with new infection.

The widespread use of antiretroviral drugs has led to the development and subsequent transmission of drug-resistant HIV-1 and the transmission of drug-resistant viruses has been documented through vertical, sexual, and parenteral routes [36-38]. Patients who are infected with drug-resistant HIV-1 and initiate antiretroviral therapy show poorer treatment responses than patients who are infected with wild-type (WT) viruses [34, 35]. Also, in the absence of selection pressures exerted by drugs, some transmitted drug-resistance mutations may persist for months before reversion to a more replication-competent variant. Even when these drug resistant mutations are no longer detectable by population-based nucleotide-sequence, they can persist in the reservoir of latently infected CD4+ memory $\mathrm{T}$ cells and may rapidly reemerge under the selective pressure provided by antiretroviral treatment [39, 40]. In subjects who acquired drug-resistant virus during primary infection, plasma HIV RNA is not suppressed as readily by potent antiretroviral therapy. The slower response to the treatment and the limited viral suppression may facilitate the selection of variants with greater drug resistance.

Thus, given the current spread and changing trends in HIV epidemiology in China, it is extremely urgent to understand the prevalence of drug-resistant strains in China and its changing patterns over time. Otherwise, we will face insurmountable challenges in tailoring our ARV regimens to elicit optimal therapeutic responses.

\section{CONCLUSIONS}

It is hard to predict the future of HIV epidemic will shape-up, since a number of complicating factors appear to unfold, including the possible effects of government intervention and unexpected changes (for the better or for the worse) in the behavior of affected populations. However, it is likely that the number of HIV infections is now on the rise. It is expected that the total number of HIV infections in China will reach one million, and that this number could rise to 10 million before 2010, if no effective countermeasures are taken. The hope is that with a timely and effective response it may be possible to keep that number much lower, perhaps not exceeding 1.5 million.

China stands at a critical juncture to prevent widespread of HIV transmission from the high risk groups to the general population. Comprehensive approaches are necessary, 
integrating prevention and treatment efforts. Government, $\mathrm{NGO}$, and international organizations bear responsibility to stop this epidemic in China. Scientific communities and pharmaceutical companies both inside and outside China need to work jointly to develop more potent anti-HIV drugs and therapeutics to inhibit viral replication and reduce HIV transmission. We have seen clear evidence in favor of evolution of complex second and third generation recombinant viruses. Continued monitoring and surveillance of these viruses is needed, if an HIV vaccine is to be developed. Moreover, concerted efforts by joint ventures between the state and the private sector are highly needed for developing an HIV vaccine for the ultimate control of HIV and its spread in the most populous nation of the world.

\section{REFERENCES}

1 National Intelligence Council. The Next Wave of HIV/AIDS: Nigeria, Ethiopia, Russia, India, and China. 2002. http://www. fas.org/irp/nic/hiv-aids.html.

2 UNAIDS. UNAIDS China report. 2005. http://www.unaids. org.

3 Zeng Y, Fan J, Zhang Q, et al. Detection of antibody to LAV/ HTLV-III in sera from hemophiliacs in China. AIDS Res 1986; 2 Suppl 1:S147-9.

4 Piyasirisilp S, McCutchan FE, Carr JK, et al. A recent outbreak of human immunodeficiency virus type 1 infection in southern China was initiated by two highly homogeneous, geographically separated strains, circulating recombinant form $\mathrm{AE}$ and a novel BC recombinant. J Virol 2000; 74:11286-95.

5 Sun X, Nan J, Guo Q. AIDS and HIV infection in China. AIDS 1994; 8 Suppl 2:S55-9.

6 Xia M, Kreiss JK, Holmes KK. Risk factors for HIV infection among drug users in Yunnan province, China: association with intravenous drug use and protective effect of boiling reusable needles and syringes. AIDS 1994; 8:1701-6.

7 Yang R, Xia X, Kusagawa S, et al. On-going generation of multiple forms of HIV-1 intersubtype recombinants in the Yunnan Province of China. AIDS 2002; 16:1401-7.

8 Avert ed. HIV and AIDS in China. 2005. http://www.avert.org/ aidschina.htm

9 Zhang KL, Ma SJ. Epidemiology of HIV in China. BMJ 2002; 324:803-4.

10 China Ministry of Health and the UN Theme Group on HIV/ AIDS in China. China Responds to AIDS: HIV/AIDS Situation and Needs Assessment Report. 1997.

11 Beyrer C, Razak MH, Lisam K, et al. Overland heroin trafficking routes and HIV-1 spread in south and south-east Asia. AIDS 2000; 14:75-83.

12. Zheng $\mathrm{X}$, Tian $\mathrm{C}$, Choi KH, et al. Injecting drug use and HIV infection in southwest China. AIDS 1994; 8:1141-7.

13 Kaufman J, Jing J. China and AIDS - the time to act is now. Science 2002; 296:2339-40.

14 Su B, Liu L, Wang F, et al. HIV-1 subtype B' dictates the AIDS epidemic among paid blood donors in the Henan and Hubei provinces of China. AIDS 2003; 17:2515-20.

15 Carr JK, Foley BT, Leitner T, et al. Reference Sequences Representing the Principal Genetic Diversity of HIV-1 in the Pandemic., in Human Retroviruses and AIDS 1998, K.C. Korber B, Foley B, Hahn B, McCutchan F, Mellors JW, and Sodroski J., Editor. 1998, Theoretical Biology and Biophysics Group: Los Alamos, NM. p. III-10-19.

16 McCutchan FE, Hegerich PA, Brennan TP, et al. Genetic variants of HIV-1 in Thailand. AIDS Res Hum Retroviruses 1992; 8: 1887-95.

17 Weniger BG, Takebe Y, Ou CY, Yamazaki S. The molecular epidemiology of HIV in Asia. AIDS 1994; 8 Suppl 2:S13-28.

18. Tovanabutra S, Beyrer C, Sakkhachornphop S, et al. The changing molecular epidemiology of HIV type 1 among northern Thai drug users, 1999 to 2002. AIDS Res Hum Retroviruses 2004; 20: 465-75.

19. Yang R, Kusagawa S, Zhang C, et al. Identification and characterization of a new class of human immunodeficiency virus type 1 recombinants comprised of two circulating recombinant forms, CRF07_BC and CRF08_BC, in China. J Virol 2003; 77:685-95.

20. Graf M, Shao Y, Zhao Q, et al. Cloning and characterization of a virtually full-length HIV type 1 genome from a subtype B'-Thai strain representing the most prevalent B-clade isolate in China. AIDS Res Hum Retroviruses 1998; 14:285-8.

21 Luo CC, Tian C, Hu DJ, et al. HIV-1 subtype C in China. Lancet 1995; 345:1051-2.

$22 \mathrm{Su} \mathrm{L}$, Graf M, Zhang Y, et al. Characterization of a virtually fulllength human immunodeficiency virus type 1 genome of a prevalent intersubtype $\left(\mathrm{C} / \mathrm{B}^{\prime}\right)$ recombinant strain in China. J Virol 2000; 74:11367-76.

23 UNAIDS/WHO. AIDS epidemic update, December 2004. 2004. http://www.unaids.org.

24 Lee WM. Hepatitis B virus infection. N Engl J Med 1997; 337: 1733-45.

25 Thio CL, Seaberg EC, Skolasky R Jr, et al. HIV-1, hepatitis B virus, and risk of liver-related mortality in the Multicenter Cohort Study (MACS). Lancet 2002; 360:1921-6.

26 Levine OS, Vlahov D, Brookmeyer R, Cohn S, Nelson KE. Differences in the incidence of hepatitis $\mathrm{B}$ and human immunodeficiency virus infections among injecting drug users. J Infect Dis 1996; 173:579-83.

27 Martin-Carbonero L, Soriano V, Valencia E, et al. Increasing impact of chronic viral hepatitis on hospital admissions and mortality among HIV-infected patients. AIDS Res Hum Retroviruses 2001; 17:1467-71.

28 Shepard CW, Finelli L, Alter MJ. Global epidemiology of hepatitis $C$ virus infection. Lancet Infect Dis 2005; 5:558-67.

29 Zhang L, Chen Z, Cao Y, et al. Molecular characterization of human immunodeficiency virus type 1 and hepatitis $C$ virus in paid blood donors and injection drug users in china. J Virol 2004; 78:13591-9.

30 Ruan YH, Hong KX, Liu SZ, et al. Community-based survey of $\mathrm{HCV}$ and HIV coinfection in injection drug abusers in Sichuan Province of China. World J Gastroenterol 2004; 10:1589-93.

31 Zhang C, Yang X, Xia X, et al. High prevalence of HIV-1 and hepatitis $\mathrm{C}$ virus coinfection among injection drug users in the southeastern region of Yunnan, China. J Acquir Immune Defic Syndr 2002; 29:191-6.

32 Zhang M, Sun XD, Mark SD, et al. Hepatitis C virus infection, Linxian, China. Emerg Infect Dis 2005; 11:17-21.

33 Boden D, Hurley A, Zhang L, et al. HIV-1 drug resistance in newly infected individuals. JAMA 1999; 282:1135-41. 
34 Grant RM, Hecht FM, Warmerdam M, et al. Time trends in primary HIV-1 drug resistance among recently infected persons. JAMA 2002; 288:181-8.

35 Little SJ, Holte S, Routy JP, et al. Antiretroviral-drug resistance among patients recently infected with HIV. N Engl J Med 2002; 347:385-94.

36 Erice A, Mayers DL, Strike DG, et al. Brief report: primary infection with zidovudine-resistant human immunodeficiency virus type 1. N Engl J Med 1993; 328:1163-5.

37 Masquelier B, Lemoigne E, Pellegrin I, et al. Primary infection with zidovudine-resistant HIV. N Engl J Med 1993; 329:1123-4.

38 Veenstra J, Schuurman R, Cornelissen M, et al. Transmission of zidovudine-resistant human immunodeficiency virus type 1 vari- ants following deliberate injection of blood from a patient with AIDS: characteristics and natural history of the virus. Clin Infect Dis 1995; $21: 556-60$.

39 Wong JK, Hezareh M, Gunthard HF, et al. Recovery of replication-competent HIV despite prolonged suppression of plasma viremia. Science 1997; 278:1291-5.

40 Finzi D, Hermankova M, Pierson T, et al. Identification of a reservoir for HIV-1 in patients on highly active antiretroviral therapy. Science 1997; 278:1295-300.

41 Gianotti N, Boeri E, Maillard M, et al. In vivo dynamics of the $103 \mathrm{~N}$ mutation following the withdrawal of non-nucleoside reverse transcriptase inhibitors in HIV-infected patients: preliminary results. New Microbiol 2004; 27:111-7. 